\title{
Prospects of Forming High-Spin Polar Molecules from Ultracold Atoms
}

\author{
Matthew D. Frye $\odot,{ }^{1}$ Simon L. Cornish $\odot{ }^{2}$, and Jeremy M. Hutson $\oplus^{1, *}$ \\ ${ }^{1}$ Joint Quantum Centre (JQC) Durham-Newcastle, Department of Chemistry, \\ Durham University, South Road, Durham, DH1 3LE, United Kingdom \\ ${ }^{2}$ Joint Quantum Centre (JQC) Durham-Newcastle, Department of Physics, \\ Durham University, South Road, Durham, DH1 3LE, United Kingdom
}

(Received 21 October 2019; revised 12 March 2020; accepted 22 July 2020; published 7 October 2020)

\begin{abstract}
We investigate Feshbach resonances in collisions of high-spin atoms such as Er and Dy with closed-shell atoms such as $\mathrm{Sr}$ and $\mathrm{Yb}$, using coupled-channel scattering and bound-state calculations. We consider both low-anisotropy and high-anisotropy limits. In both regimes, we find many resonances with a wide variety of widths. The wider resonances are suitable for tuning interatomic interactions, while some of the narrower resonances are highly suitable for magnetoassociation to form high-spin molecules. These molecules might be transferred to short-range states, where they would have large magnetic moments and electric dipole moments that can be induced with very low electric fields. The results offer the opportunity to study mixed quantum gases where one species is dipolar and the other is not and open up important prospects for a new field of ultracold high-spin polar molecules.
\end{abstract}

DOI: $10.1103 /$ PhysRevX.10.041005

Subject Areas: Atomic and Molecular Physics, Quantum Physics

\section{INTRODUCTION}

Magnetic Feshbach resonances play a crucial role in the study of ultracold atoms [1]. They can be used to control interaction strengths by varying the scattering length [2]. This tunability has opened up many applications in the many-body physics of strongly interacting systems [3]. Tunable Feshbach resonances also form the basis of magnetoassociation [4] and are crucial in the formation of ultracold ground-state molecules composed of alkalimetal atoms [5-10]. These molecules are now opening up new areas of research into dipolar physics [11-14], quantum simulation and computation [15-18], and controlled chemistry [19-21].

There is now much interest in creating ultracold molecules from different species and with different properties. In particular, molecules with both electric and magnetic dipole moments will open up new possibilities for designing quantum many-body systems [22,23] and for tests of fundamental symmetries [24]. In this context, there has been significant work toward producing ultracold ${ }^{2} \Sigma$ molecules, both by direct laser cooling [25-28] and by association of alkali-metal and closed-shell atoms [29-34].

*j.m.hutson@durham.ac.uk

Published by the American Physical Society under the terms of the Creative Commons Attribution 4.0 International license. Further distribution of this work must maintain attribution to the author(s) and the published article's title, journal citation, and DOI.
However, the former method still produces only relatively low densities and is highly system specific, while the latter method is experimentally challenging due to the sparse and very narrow Feshbach resonances in such systems.

In this paper, we investigate Feshbach resonances in collisions of lanthanide atoms with large orbital angular momentum $l$, such as $\operatorname{Er}\left({ }^{3} \mathrm{H}_{6}\right)$ or Dy $\left({ }^{5} \mathrm{I}_{8}\right)$, with closed-shell $\left({ }^{\mathrm{I}} \mathrm{S}\right)$ atoms, such as $\mathrm{Yb}$ or $\mathrm{Sr}$. All four of these elements can be cooled to ultracold temperatures [35-41] and have numerous abundant isotopes including both bosons and fermions. The large masses result in high densities of molecular states near threshold. These states produce many resonances with a wide variety of widths. Some of them will be suitable for forming molecules by magnetoassociation, and others will be suitable for tuning interactions in atomic mixtures.

The variety of isotopes available should allow the formation of both bosonic and fermionic molecules. These molecules will inherit the large magnetic moments of Er and Dy. It should be possible to transfer them to shortrange states using stimulated Raman adiabatic passage (STIRAP), as has been achieved for alkali-metal dimers [5-10]. In these short-range states, they are likely to have substantial electric and magnetic dipole moments. The magnetic dipole interactions will be far larger than for currently available ultracold molecules, and systems built from the molecules may exhibit new types of phase transition resulting from the competition between electric and magnetic interactions.

The short-range states are characterized by the projection $\Omega$ of the electronic (orbital and spin) angular momentum along the internuclear axis. For molecules in states with 
$|\Omega|>0$, the existence of small $\Omega$-type doubling splittings allows the electric dipole moment to be oriented with small electric fields [42]. This is a particular benefit for the high-spin molecules considered here, since $\Omega$-doubling splittings decrease fast as $|\Omega|$ increases. Such molecules are analogous to symmetric tops; they will allow the simulation of important problems in quantum magnetism $[43,44]$ and may also open up new opportunities for scalable quantum computers [45]. These advantages are not offered by molecules formed from pairs of atoms in $\mathrm{S}$ states, even if they have high spin [46], since there is no $\Omega$ doubling for $\Sigma$ states.

The Feshbach resonances will also allow the study of quantum-degenerate Bose-Bose, Bose-Fermi, and FermiFermi mixtures with tunable interactions. These mixtures will have the new feature that one component interacts by dipole-dipole interactions, while the other does not. The mixtures are expected to exhibit rich phase behavior, including superfluidity, supersolidity [47], and formation of bound dipolar droplets [48]. They will open up new possibilities for the study of imbalanced Fermi gases, which may exhibit exotic phase behavior and allow the exploration of new pairing mechanisms [49-51].

\section{THEORETICAL METHODS AND INTERACTION POTENTIALS}

The collision Hamiltonian for any pair of atoms $A$ and $X$ may be written

$$
\frac{\hbar^{2}}{2 \mu}\left(-\frac{d^{2}}{d R^{2}}+\frac{\hat{L}^{2}}{R^{2}}\right)+\hat{h}_{A}+\hat{h}_{X}+\hat{V}(R),
$$

where $R$ is the interatomic distance, $\mu$ is the reduced mass, and $\hat{L}$ is the angular momentum operator for the relative motion. $\hat{h}_{A}$ and $\hat{h}_{X}$ are the Hamiltonians of the separated atoms [52], including external fields if necessary, and $\hat{V}(R)$ is the interaction operator.

In the present work, we consider the closed-shell atom $A$ to be structureless, so set $\hat{h}_{A}=0$. The states of heavy open-shell atoms such as the lanthanides Er and Dy are generally best represented using $j$ - $j$ coupling rather than Russell-Saunders coupling, but here we are interested principally in the lowest spin-orbit component of the ground state, with the maximum total angular momentum $j$ allowed by the orbital configuration. Other atomic states (even excited spin-orbit states) are too high in energy to produce resonances and play little role. We therefore choose to represent the atomic states with quantum numbers $l$ and $s$ for the total orbital and spin angular momenta, with corresponding operators $\hat{l}$ and $\hat{s}$, and the atomic Hamiltonian as

$$
\hat{h}_{X}=a_{X}^{\mathrm{so}} \hat{l} \cdot \hat{s}+\left(g_{l} \hat{l}_{z}+g_{s} \hat{s}_{z}\right) \mu_{B} B,
$$

where $B$ is the magnetic field oriented along the $z$ axis and $g_{s}$ and $g_{l}$ are the electron spin and orbital $g$ factors, respectively, both defined to be positive. We take $a_{\mathrm{Dy}}^{\text {so }}=-516.779 \times$ $h c \mathrm{~cm}^{-1}$ and $a_{\mathrm{Er}}^{\mathrm{so}}=-1159.7215 \times h c \mathrm{~cm}^{-1}$, which reproduce the splitting of the lowest two spin-orbit states [53]. In general, there can also be hyperfine terms in the single-atom Hamiltonians, but, for simplicity, we focus on isotopes without nuclear spin in this paper; we choose ${ }^{164} \mathrm{Dy}$, ${ }^{174} \mathrm{Yb},{ }^{166} \mathrm{Er}$, and ${ }^{88} \mathrm{Sr}$, which are the most abundant isotopes.

The interaction operator $\hat{V}(R)$ is a function of both orbital and spin coordinates. It can, in principle, depend on an external field, but any such dependence is neglected here. We choose to write it in the form

$$
\hat{V}(R)=\hat{V}_{\text {space }}(R)+\hat{V}_{\text {spin }}(R),
$$

where $\hat{V}_{\text {space }}(R)$ contains all terms independent of the spin degrees of freedom. Because of cylindrical symmetry, $\hat{V}_{\text {space }}(R)$ is diagonal in $\lambda$, the projection of $l$ onto the internuclear axis. We use the resolution of the identity $1=\sum_{l, \lambda}|l \lambda\rangle\langle l \lambda|$. Atomic states with $l \neq 6$ for Dy or $l \neq 5$ for Er are too high in energy to contribute, so we include only a single value of $l$ in each case, and the expansion reduces to

$$
\hat{V}_{\text {space }}(R)=\sum_{\lambda}|l \lambda\rangle\langle l \lambda| V_{\lambda}(R),
$$

where $\lambda$ takes values from $-l$ to $l$ and $V_{\lambda}(R)$ is independent of the sign of $\lambda$. The differences between the potential curves $V_{\lambda}(R)$ may be viewed as anisotropy in $\hat{V}_{\text {space }}(R)$. The operator $\hat{V}_{\text {spin }}(R)$ might include terms such as the dependence of $a_{X}^{\text {so }}$ on $R$; for simplicity, we neglect it here, although it may be needed when making quantitative comparisons between experiment and theory at a later stage.

Very little is known about the interactions in the systems we consider here. However, we can make reasonable estimates on physical grounds. The attractive parts of the potentials are likely to be dominated by dispersion forces, with little contribution from chemical bonding, because the outermost $s$ orbitals are filled and the partially filled $f$ shells of the lanthanides are submerged. At long range, the interaction potentials are of the form $V(R)=-C_{6} / R^{6}$. In physical terms, differences between the potentials $V_{\lambda}(R)$ may come from differences in either the attractive or the repulsive part. To model these separately, we represent the interaction potentials in Lennard-Jones form

$$
\begin{aligned}
V_{\lambda}(R) & =C_{12, \lambda} R^{-12}-C_{6, \lambda} R^{-6} \\
& =D_{e, \lambda}\left[\left(\frac{R_{e, \lambda}}{R}\right)^{12}-2\left(\frac{R_{e, \lambda}}{R}\right)^{6}\right],
\end{aligned}
$$

where $C_{12, \lambda}$ is the repulsive coefficient and $D_{e, \lambda}$ is the well depth at equilibrium distance $R_{e, \lambda}$. More sophisticated 
forms of $V_{\lambda}(R)$ could be used but would not change the qualitative conclusions.

We obtain parameters for $\mathrm{Er}+\mathrm{Sr}$ and $\mathrm{Dy}+\mathrm{Yb}$ from combination rules based on the interaction potentials for the corresponding homonuclear systems. Values of the $C_{6}$ coefficients for all the homonuclear systems have been obtained experimentally [54,55] or theoretically [56-59]. We obtain values of the isotropic coefficients $C_{6}^{(0)}$ for $\mathrm{Er}+\mathrm{Sr}$ and $\mathrm{Dy}+\mathrm{Yb}$ from Tang's combination rule [60], for which we use dispersion coefficients from Refs. [54,55,58,59] and the atomic polarizabilities from Ref. [61], giving 2092 and $2359 E_{h} a_{0}^{6}$, for Dy $+\mathrm{Yb}$ and $\mathrm{Er}+\mathrm{Sr}$, respectively.

Er and Dy have tensor and vector polarizabilities that result in anisotropic $C_{6}$ coefficients. The ratio of the anisotropic coefficient $C_{6}^{(2)}$ to the isotropic one is comparable to the ratio of the tensor and scalar static polarizabilities of the open-shell atom; values for this range from 0.016 [62] to 0.018 [58] for Er and from 0.005 [59] to 0.026 [62] for Dy. We base our main calculations on a ratio $C_{6}^{(2)} / C_{6}^{(0)}=0.017$ for both systems but explore the effects of variations.

The interaction potential for $\mathrm{Sr}+\mathrm{Sr}$ has been studied in detail spectroscopically; the well depth and equilibrium distance are known precisely to be $1081.64 \mathrm{~cm}^{-1}$ and $4.672 \AA$, respectively, and the potential supports 63 vibrational levels for ${ }^{88} \mathrm{Sr}[54,63]$. For $\mathrm{Yb}+\mathrm{Yb}$, there is considerable variation in the well depth between different levels of theory [64], but the dependence of the nearthreshold bound states on the isotopic mass shows that it supports 72 vibrational levels $[55,65]$. We base our calculations on the depth $739.73 \mathrm{~cm}^{-1}$ obtained by Borkowski et al. [55]. Petrov, Tiesinga, and Kotochigova [66] carried out electronic structure calculations of one component of the potential for Dy + Dy and obtained a well depth of $785.7 \mathrm{~cm}^{-1}$. To obtain interaction potentials for $\mathrm{Er}+\mathrm{Sr}$ and $\mathrm{Dy}+\mathrm{Yb}$, we estimate the isotropic well depths $D_{e}$ as the geometric mean of those for the two homonuclear systems. For this, we estimate the well depth for $\mathrm{Er}+\mathrm{Er}$ as $471.1 \mathrm{~cm}^{-1}$, obtained from the well depth for Dy + Dy [66] scaled by the square of the ratio of the $C_{6}$ coefficients. The resulting well depths for the heteronuclear systems are about $760 \mathrm{~cm}^{-1}$ for Dy $+\mathrm{Yb}$ and $710 \mathrm{~cm}^{-1}$ for $\mathrm{Er}+\mathrm{Sr}$.

To model the effects of long-range anisotropies, we choose a set of coefficients $C_{6, \lambda}$ that correspond to the required value of $C_{6}^{(2)}$,

$$
C_{6, \lambda}=C_{6}^{(0)}+C_{6}^{(2)} g_{2}(l, \lambda)
$$

where $g_{k}(l, \lambda)=(-1)^{\lambda}(2 l+1)\left(\begin{array}{lll}l & k & l \\ 0 & 0 & 0\end{array}\right)\left(\begin{array}{ccc}l & k & l \\ -\lambda & 0 & \lambda\end{array}\right)$.

We obtain an isotropic repulsive coefficient from

$$
C_{12}^{(0)}=\left(C_{6}^{(0)}\right)^{2} / 4 D_{e} .
$$

We fix $C_{12, \lambda}$ at $C_{12}^{(0)}$ for all $\lambda$, so that $D_{e, \lambda}$ is different for each $\lambda$.

Short-range anisotropies are less well understood. They might be much stronger because of the effects of higherorder dispersion, chemical bonding, or repulsive forces. To model these effects, we choose a set of well depths $D_{e, \lambda}$ and fix $C_{6}$ at its isotropic value, so that $C_{12, \lambda}$ is different for each $\lambda$. The isotropic potential curves described above support 64 (52) vibrational levels for $\mathrm{Dy}+\mathrm{Yb}(\mathrm{Er}+\mathrm{Sr})$. Because of this, a $3.1 \%$ (3.8\%) scaling of the potential is sufficient to shift the scattering length through a complete cycle [67]. It thus seems likely that the scattering lengths for different values of $\lambda$ are essentially random. Varying $D_{e}$ with $C_{6}$ unchanged is not a simple scaling, but we nevertheless find that a scaling of $D_{e}$ by $4.7 \%$ (5.6\%) produces a full cycle in the scattering length. Our strategy is, thus, to choose values of $D_{e, \lambda}$ randomly from a range from 0.93 to 1.07 (0.91 to $1.09)$ times the starting value. This range is chosen so that even the isotropic potential [essentially, the mean $V_{\lambda}(R)$ ] is random. This produces anisotropies of higher order than the dispersion anisotropy above, with $k>2$ in Eq. (7). It should be emphasized that this choice probably represents the maximum coupling that is likely to arise from shortrange anisotropy, and the coupling may turn out to be weaker.

We perform scattering calculations using the MOLSCAT package $[68,69]$ and bound-state calculations with the BOUND package $[68,70]$. Both packages use coupledchannel methods, in which the total wave function is expanded in a basis set for all coordinates except the interatomic distance $R$. This gives a set of coupled differential equations with respect to $R$. The methods are similar to those of Refs. [71,72] but without terms that correspond to $\hat{h}_{A}$. We use a partially coupled basis set $\left|(l s) j m_{j}\right\rangle\left|L M_{L}\right\rangle$, where $m_{j}$ and $M_{L}$ are the projections of $j$ and $L$, respectively, onto the axis of the magnetic field (the $z$ axis). Matrix elements of the interaction potential are calculated as in Ref. [73]. The only conserved quantum numbers are the projection of the total angular momentum onto the $z$ axis, $M_{\text {tot }}=m_{j}+M_{L}$, and the total parity $(-1)^{l+L}$. Since the atomic spin-orbit splittings for both Dy and Er are substantially larger than the potential well depths, we restrict our basis functions to those for the lowest spin-orbit state, with $j=l+s$. This restriction means that the spin-orbit coupling in $\hat{h}_{X}$ has no effect except to shift the whole system by a constant energy. We include all functions with $L$ up to $L_{\max }$. For the case of 
long-range anisotropy, the basis set converges quickly with respect to $L$, and we choose $L_{\max }=10$. For short-range anisotropy, which can be stronger, we include basis functions up to $L_{\max }=2 j$. This is because, in a representation where $j$ and $L$ are coupled to form a total angular momentum $J$, the $s$-wave channel $(L=0)$ at the lowest threshold corresponds to $J=j$; representing all states of this total $J$ requires functions up to $L=2 j$. In the resulting calculations, the widest resonances are well converged. For strong anisotropy, adding higher- $L$ basis functions shifts some of the narrower resonances and adds new narrow ones.

The coupled equations are solved using log-derivative propagators. For scattering calculations, the log-derivative matrix is propagated outward from $R_{\min }=2.5 \AA$ to $R_{\text {mid }}=20 \AA$ using the diabatic log-derivative method of Manolopoulos $[74,75]$ with a fixed step size of $0.005 \AA$ and then from $R_{\operatorname{mid}}$ to $R_{\max }=2000 \AA$ using the log-derivative Airy propagator of Alexander and Manolopoulos with a variable step size [76,77]. The solution is matched to asymptotic boundary conditions at $R_{\max }$ to obtain the scattering matrix $\mathbf{S}$. The scattering length is calculated as $a(k)=(i k)^{-1}\left(1-S_{00}\right) /\left(1+S_{00}\right)$, where $k=\sqrt{2 \mu E} / \hbar$ is the wave vector and $S_{00}$ is the diagonal $S$-matrix element in the incoming $s$-wave channel for the chosen value of $m_{j}$. The kinetic energy $E$ in the incoming channel is set to $100 \mathrm{nK}$. For bound-state calculations, one log-derivative matrix is propagated outward from $R_{\min }$ to $R_{\text {mid }}$, and another is propagated inward from $R_{\max }$ to $R_{\text {mid }}$. BOUND finds eigenenergies by searching for the energies at which these solutions can be matched at $R_{\text {mid }}$ as described in Ref. [78].

\section{FESHBACH RESONANCES AND BOUND STATES}

A magnetic Feshbach resonance occurs when a bound state is tuned across a scattering threshold with a magnetic field $B$. Molecules may be formed by sweeping the field across a resonance, which is referred as magnetoassociation. Resonances may also be used to control interactions by tuning the scattering length.

For an isolated resonance at the lowest threshold, the scattering length $a(B)$ has a characteristic pole at the resonance position $B_{\text {res }}$ [2],

$$
a(B)=a_{\mathrm{bg}}\left(1-\frac{\Delta}{B-B_{\mathrm{res}}}\right),
$$

where $a_{\mathrm{bg}}$ is the background scattering length and $\Delta$ is the resonance width.

We carry out coupled-channel calculations to characterize Feshbach resonances at the lowest threshold of Dy + $\mathrm{Yb}\left({ }^{5} \mathrm{I}_{8}, m_{j}=-8\right)$. We first consider interaction potentials with a purely long-range anisotropy characterized by $C_{6}^{(2)} / C_{6}^{(0)}=0.017$. The details of the results depend on the scattering length $a_{\text {iso }}$ of the isotropic potential, which is at present unknown. We, therefore, perform calculations for 100 different interaction potentials, with values of $C_{12}^{(0)}$ that vary over a range of approximately $4.7 \%$, covering the full

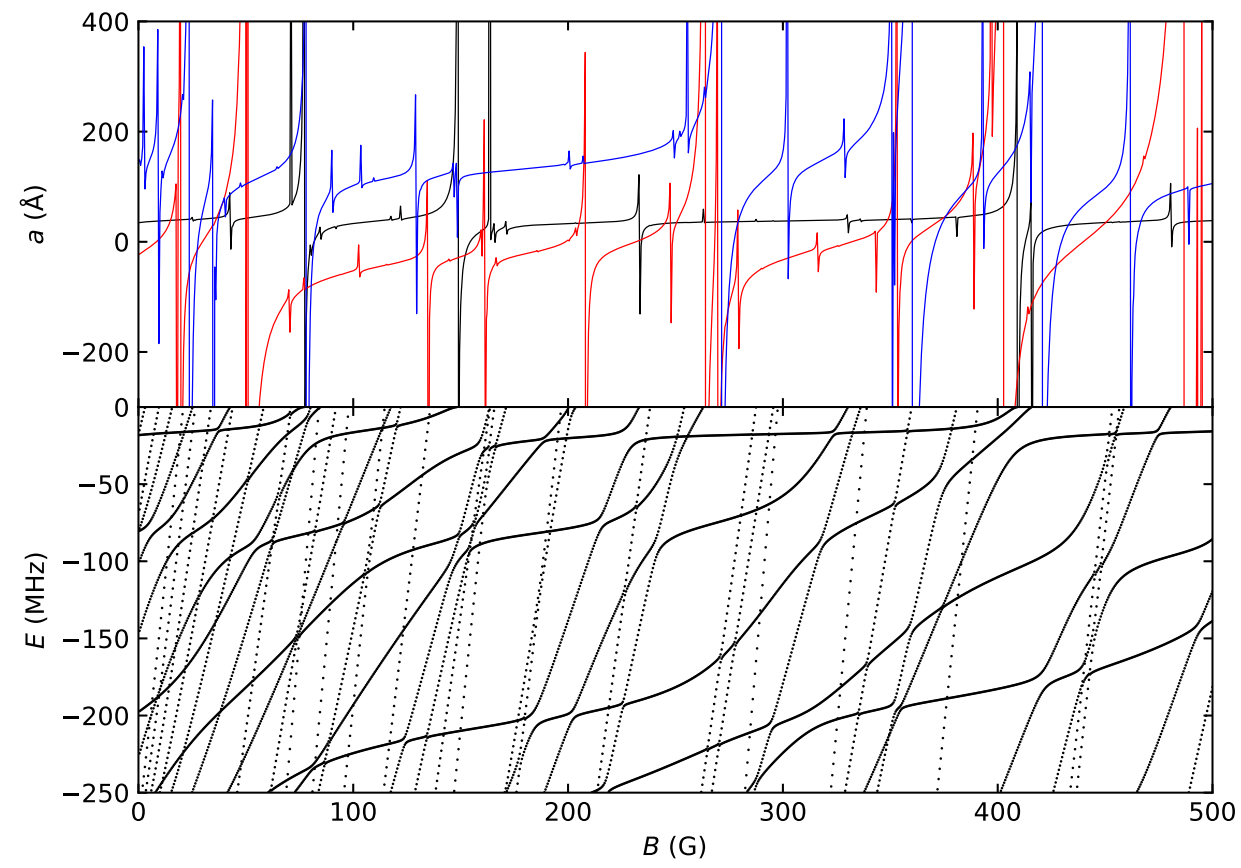

FIG. 1. Top: Scattering lengths as a function of the magnetic field for Dy + Yb with long-range anisotropy $C_{6}^{(2)} / C_{6}^{(0)}=0.017$ for three different values of the scattering length $a_{\text {iso }}=54$ (black lines), -80 (red lines), and $168 \AA$ (blue lines). Bottom: The corresponding nearthreshold bound states for $a_{\text {iso }}=54 \AA$. 
range of $a_{\text {iso }}$ but with similar well depths. The upper panel in Fig. 1 shows the $s$-wave scattering length for three representative potentials with $a_{\text {iso }}=54,-80$, and $168 \AA$.

We characterize all the Feshbach resonances that exist below $500 \mathrm{G}$ for each interaction potential, using the method of Frye and Hutson $[79,80]$. There are typically 50 resonances for each potential, and we obtain values of $B_{\text {res }}, \Delta$, and $a_{\mathrm{bg}}$ for each resonance. It should be noted that the resonance width $\Delta$ can be artificially large if $a_{\mathrm{bg}}$ is particularly small. A better measure of the strength of the resonant pole, which retains dimensions of magnetic field, is the normalized width [34]

$$
\bar{\Delta}=a_{\mathrm{bg}} \Delta / \bar{a},
$$

where $\bar{a}=\left(2 \mu C_{6} / \hbar^{2}\right)^{1 / 4} \times 0.4779888 \ldots$ is the mean scattering length of Gribakin and Flambaum [81] and is $40.3 \AA$ for ${ }^{164} \mathrm{Dy}+{ }^{174} \mathrm{Yb}$ and $37.7 \AA$ for ${ }^{166} \mathrm{Er}+{ }^{88} \mathrm{Sr}$.

All the interaction potentials produce many Feshbach resonances with a wide distribution of widths. The widest resonances are due to bound states with predominantly $L=2$ character, and the narrower ones to states with predominantly $L=4$ character. However, as seen below, there is substantial mixing between states of different $L$. Very narrow resonances with $L>4$ exist, and some of them are just visible in Fig. 1. In Supplemental Material [82], we plot the number of resonances with $\bar{\Delta}>\bar{\Delta}_{\text {min }}$ as a function of $\bar{\Delta}_{\text {min }}: 95 \%$ of the interaction potentials with $C_{6}^{(2)} / C_{6}^{(0)}=0.017$ have at least ten resonances with $\bar{\Delta}>0.1 \mathrm{G}$ at fields below $500 \mathrm{G}$.

The details of the resonances depend on $a_{\text {iso }}$, but choosing a different value of $C_{12}^{(0)}$ that produces a different number of bound states but the same $a_{\text {iso }}$ produces very similar results. Most resonances shift by less than $0.2 \mathrm{G}$, and their widths change by less than $20 \%$.

The lower panel in Fig. 1 shows the near-threshold bound states responsible for the resonances in the upper panel for the case of $a_{\text {iso }}=54 \AA$. The near-horizontal line near $E=-20 \mathrm{MHz}$ is due to a state that is predominantly $L=0, m_{j}=-8$ in character. This state has broad avoided crossings with $L=2$ states and much narrower ones with $L=4$ states; the slopes of these states increase and the strengths of the crossings decrease as $m_{j}$ increases from -8 . More generally, there are strong crossings due to direct interactions between pairs of states with $\Delta L \leq 2$ and $\Delta m_{j} \leq 2$ and weaker crossings due to higher-order interactions otherwise. For $\mathrm{Dy}+\mathrm{Yb}$, the characteristic energy $\bar{E}=\hbar^{2} /\left(2 \mu \bar{a}^{2}\right)=h \times 3.69 \mathrm{MHz}$. There is a bound state with $L=0$ (2) in the top $36.1 \bar{E}(93.4 \bar{E})$ of the well for any value of $a_{\text {iso }}$ [83], so in the top 133 (344) MHz here. The $g$ factor for the ${ }^{5} \mathrm{I}_{8}$ state is $g_{j}=1.25$, so the relative gradient of states with $m_{j}=-8$ and -6 is $2 g_{j} \mu_{B} / h=3.50 \mathrm{MHz} / \mathrm{G}$. The lowest-field $L=2$ resonance is expected to occur at a field below $93.4 \bar{E} / 2 g_{j} \mu_{B}=98 \mathrm{G}$ for any value of $a_{\text {iso }}$.
We repeat the calculations for a smaller long-range anisotropy $C_{6}^{(2)} / C_{6}^{(0)}=0.0085$, which is half the value in Fig. 1. Representative examples of scattering lengths and bound-state energies are shown in Supplemental Material [82]. The bound-state diagram shows considerably less mixing between different states. The resonance positions change very little for the same value of $a_{\text {iso }}$, but the widths scale approximately as $\left|C_{6}^{(2)}\right|^{2}$ for resonances due to $L=2$ states and as $\left|C_{6}^{(2)}\right|^{4}$ for those due to $L=4$ states. This indicates that a long-range anisotropy of this magnitude may be viewed as operating perturbatively. In this case, all 100 potentials considered had at least three resonances with $\bar{\Delta}>0.1 \mathrm{G}$ below $500 \mathrm{G}$. The long-range anisotropies considered here probably represent approximately the minimum degree of coupling that is likely in these systems.

We carry out analogous calculations on $\mathrm{Er}+\mathrm{Sr}$. The results are qualitatively similar, and representative examples are shown in Supplemental Material [82]. In this case, $\bar{E}=h \times 6.18 \mathrm{MHz}$ and $g_{j}=1.17$, so the lowest-field $L=2$ resonance is expected to occur below $93.4 \bar{E} / 2 g_{j} \mu_{B}=$ $176 \mathrm{G}$ for any value of $a_{\text {iso }}$. The resulting densities of bound states and resonances are somewhat lower than for Dy $+\mathrm{Yb}$.

We next consider interaction potentials with short-range anisotropies, chosen as described in Sec. II. The upper panel in Fig. 2 shows the scattering length for $\mathrm{Dy}+\mathrm{Yb}$ $\left(m_{j}=-8\right)$ for a representative example, and the lower panel shows the near-threshold bound states. Further examples are shown in Supplemental Material [82]. These interaction potentials are much more strongly anisotropic than those based on long-range anisotropy above, and their higher-order anisotropies cause direct couplings with larger values of $\Delta L$ and $\Delta m_{j}$. They all produce many Feshbach resonances with a wide variety of widths. $L$ is now very poorly conserved, so the resonances are not limited to those for $L<6$. Nevertheless, it may be seen in the lower panel in Fig. 2 that there are well-defined states that maintain their character through many avoided crossings. This demonstrates that the energy-level pattern has structure even in this high-anisotropy regime.

We characterize all the resonances that exist below $500 \mathrm{G}$ for 100 different interaction potentials of this type. In Supplemental Material [82], we plot the number of resonances with $\bar{\Delta}>\bar{\Delta}_{\min }$ as a function of $\bar{\Delta}_{\min }$. We find that $95 \%$ of interaction potentials with short-range anisotropy have at least 60 resonances with $\bar{\Delta}>0.1 \mathrm{G}$ at fields below $500 \mathrm{G}$.

One advantage of these systems is the existence of many different isotopic combinations. The reduced mass may be adjusted over a range of $4.7 \%$ for $\mathrm{Dy}+\mathrm{Yb}$ or $4.6 \%$ for $\mathrm{Er}+\mathrm{Sr}$. This changes the patterns of resonances and bound states in significantly different ways in the different regimes of anisotropy. For weak anisotropy, a change in reduced mass $\mu$ is almost the same as scaling the isotropic potential; it changes the isotropic scattering length $a_{\text {iso }}$ in a smooth 


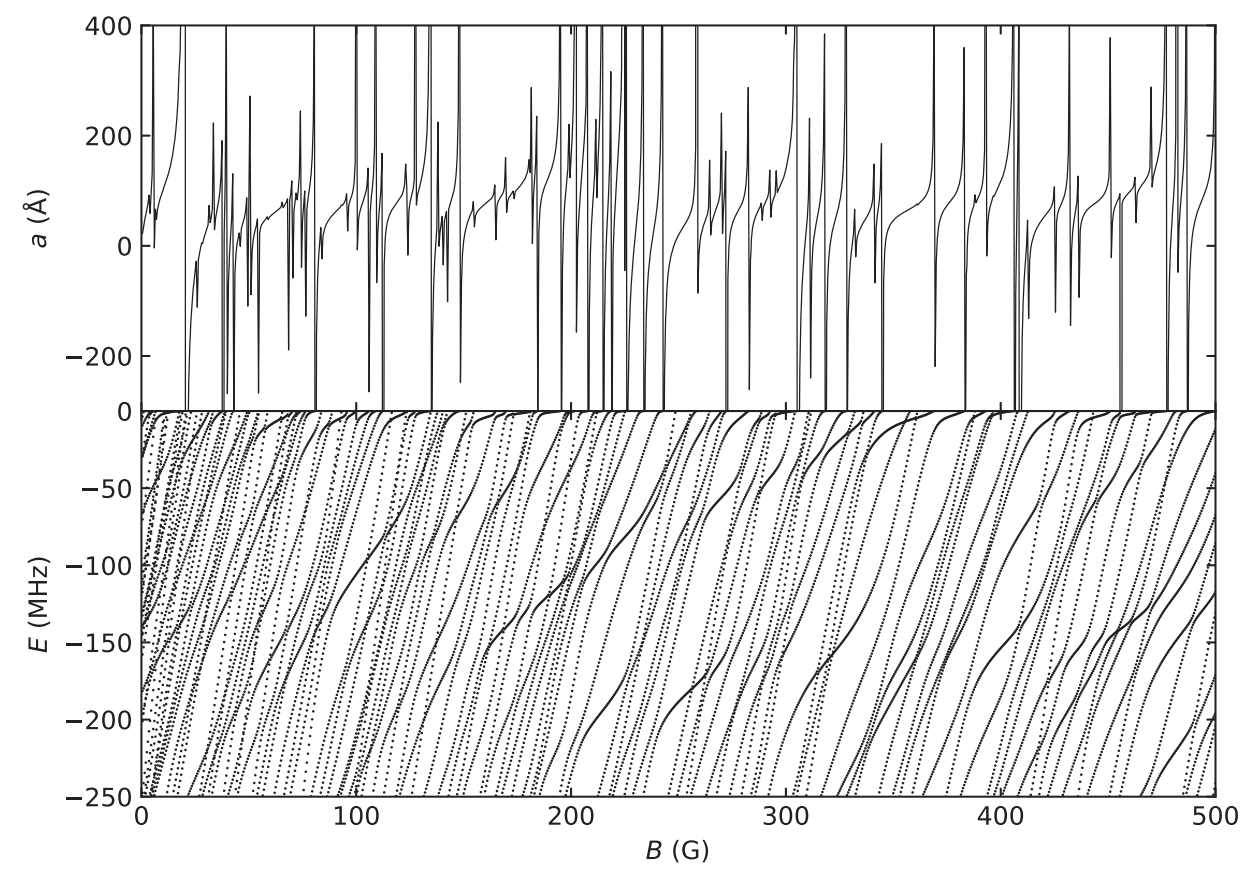

FIG. 2. Top: Scattering lengths as a function of the magnetic field for Dy + Yb with short-range anisotropy as described in the text. Bottom: The corresponding near-threshold bound states.

way. Changing the mass of the open-shell (closed-shell) atom by 2 changes $\mu$ by $0.6 \%$ ( $0.6 \%)$ for $\mathrm{Dy}+\mathrm{Yb}$ or $0.4 \%$ $(1.5 \%)$ for $\mathrm{Er}+\mathrm{Sr}$; this is sufficient to change $a_{\text {iso }}$ considerably, but it is also possible to change the isotope of both atoms to achieve a much smaller change in reduced mass. For strong anisotropy, however, the situation is different; in this case, the positions of the widest resonances shift relatively smoothly, but even a change of $0.1 \%$ in $\mu$ causes substantial changes in the relative positions of narrower resonances. Measurements on different isotopic combinations will thus provide a powerful way to distinguish between the different regimes of anisotropy.

We also calculate scattering lengths for collisions of open-shell atoms in excited Zeeman states, $m_{j}>-j$. Examples for $\mathrm{Dy}+\mathrm{Yb}$ with both long-range and shortrange anisotropies are shown in Fig. 3. Additional examples are given in Supplemental Material [82]. The scattering length is complex $(a=\alpha-i \beta)$ because of inelastic decay to lower channels. The corresponding rate coefficient for two-body inelastic decay in $s$-wave scattering is $k_{2}^{\text {inel }} \approx 2 h \beta / \mu$. The resonances show oscillations in both $\alpha$ and $\beta[79,84]$ rather than poles as at the lowest threshold. For long-range anisotropies, the resonances are relatively weakly decayed for $m_{j}=-7$, with oscillations in $\alpha$ and peaks in $\beta$ of amplitude up to $500 \AA$. The amplitudes of the oscillations decrease with increasing $m_{j}$, as the number of open channels increases. For short-range anisotropies, the decay is substantially stronger; the resonances are progressively washed out as $m_{j}$ increases, and $\beta$ approaches the value of $\bar{a}$ expected in the "universal" limit of $100 \%$ loss at short range $[85,86]$. In this case, there are visible resonances in $\alpha$ and $\beta$ at low field even for the uppermost channel with $m_{j}=j$; these arise from quasibound states trapped behind centrifugal barriers for channels with $m_{j}<j$ and $L>0$ [87].

\section{EXTENSION TO BOSE-FERMI AND FERMI-FERMI MIXTURES}

The calculations in Sec. III deal with systems where both nuclei are spin-zero bosons and the corresponding atoms are bosonic. Fermionic isotopes also exist, with nonzero nuclear spin $i$, and Bose-Fermi and Fermi-Fermi mixtures are of great interest. The fermionic isotopes include ${ }^{161} \mathrm{Dy}$ $(i=5 / 2),{ }^{163} \mathrm{Dy}(i=5 / 2),{ }^{167} \operatorname{Er}(i=7 / 2),{ }^{87} \mathrm{Sr}(i=9 / 2)$, ${ }^{171} \mathrm{Yb}(i=1 / 2)$, and ${ }^{173} \mathrm{Yb}(i=3 / 2)$. These elements have no stable bosonic isotopes with $i>0$.

Including nuclear spin introduces substantial additional complexity. Each additional spin is an extra source of angular momentum, and there are additional terms in the Hamiltonian from nuclear Zeeman effects and from couplings between the additional spin and the existing angular momenta $j$ and $L$. The cases with a nuclear spin on the lanthanide atom or on the closed-shell atom are quite different.

A nuclear spin on the closed-shell atom is uncoupled to $j$ and $L$ in the separated atoms and only weakly coupled in the molecular states that cause Feshbach resonances. A moderate magnetic field is sufficient to decouple it even for the molecule, with a well-defined projection quantum number $m_{i}$ onto the field axis. Under these circumstances, the nuclear spin acts as a spectator; the pattern of 

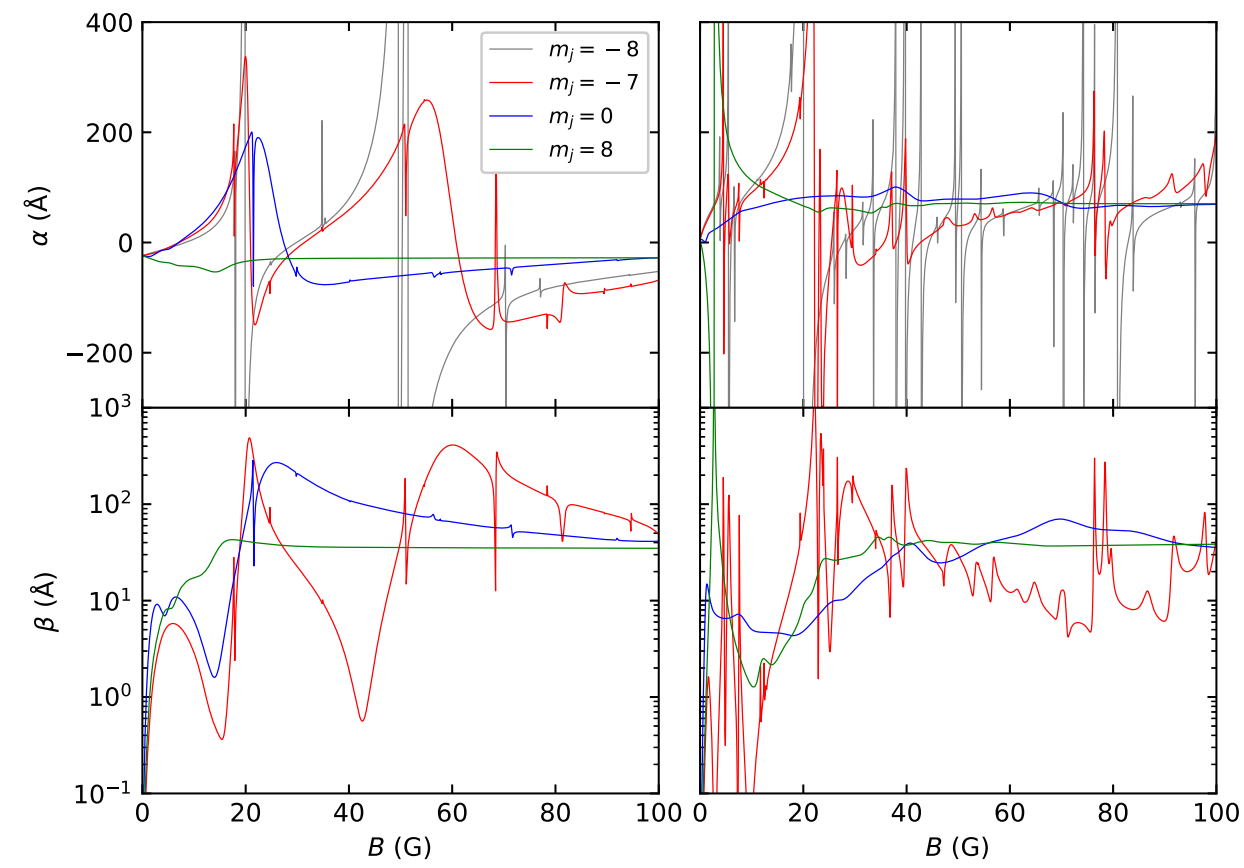

FIG. 3. Complex scattering lengths $a=\alpha-i \beta$ as a function of the magnetic field for Dy $+\mathrm{Yb}$ with Dy in excited Zeeman states $m_{j}=-7$ (red lines), 0 (blue lines), and 8 (green lines); $m_{j}=-8$ (gray lines) results are also shown for comparison. Left: Interaction potential with long-range anisotropy $C_{6}^{(2)} / C_{6}^{(0)}=0.017$ for $a_{\text {iso }}=-80 \AA$. Right: Interaction potential with short-range anisotropy as described in the text.

resonances and widths is essentially the same as without the spin, with small shifts dependent on $m_{i}$ due to nuclear Zeeman effects. There may be additional narrow resonances due to couplings involving the nuclear spin, as exist for a closed-shell atom with nuclear spin interacting with an alkali-metal atom $[33,34,88]$.

A nuclear spin on the lanthanide atom is strongly coupled to the atomic $j$ even in the separated atoms, with hyperfine splittings up to a few gigahertz $[89,90]$. There are multiple zero-field atomic states that interact and mix in a magnetic field. The states for a single value $j$ may be viewed as a more complicated version of the states of an alkali-metal atom in a magnetic field. For a high-spin atom in an $\mathrm{S}$ state, such as $\mathrm{Cr}$ or Eu [91,92], interaction with the closed-shell atom produces only a single electronic state. The resulting atomic and molecular states are then coupled only by the $R$ dependence of the hyperfine coupling constants; this coupling is relatively weak and will produce only narrow resonances, as are found for an alkali-metal atom interacting with a closed-shell atom $[29,31,33,34]$. In the present case, however, the multiple electronic states resulting from the anisotropy of the lanthanide atom play the same role as the singlet and triplet states in alkali pairs [1]. The combined effect of hyperfine coupling, Zeeman mixing, and multiple electronic states will produce complex patterns of Feshbach resonances, over and above those due to the fine structure and anisotropy alone. Characterizing these resonances in detail is beyond the scope of the present work, but they are likely to produce additional opportunities for tuning mixtures of fermionic lanthanide atoms and closed-shell atoms and producing molecules by magnetoassociation.

When there are nuclear spins on both atoms, as is the case for Fermi-Fermi mixtures of lanthanide atoms and closed-shell atoms, both the effects described above come into play, producing a rich and complex pattern of Feshbach resonances. Nevertheless, the calculations described in the present paper are likely to represent a lower bound for the number of resonances that exist.

\section{EXPERIMENTAL POSSIBILITIES}

All four atomic species considered here have been cooled to quantum degeneracy for both bosonic and fermionic isotopes. From a laser-cooling perspective, the highly magnetic lanthanide atoms are remarkably similar to their closed-shell cousins, despite their complex atomic structure. Indeed, the procedure for creating Er BoseEinstein condensates [40] was inspired by the work on $\mathrm{Yb}[35,93]$. They also have similar polarizabilities in the wavelength range commonly used for optical traps (1030$1070 \mathrm{~nm}$ ), so that cotrapping and simultaneous evaporative cooling of cotrapped samples should be straightforward. The atoms are thus highly compatible for experiments on mixtures.

The behavior of the mixtures will be complicated by the dense and chaotic intraspecies resonance structure of $\mathrm{Er}$ and Dy themselves [94-96]. However, the positions of these resonances are well documented and the majority 
have widths below $100 \mathrm{mG}$. Two possible approaches may be envisaged. Since the closed-shell atoms are unaffected by the intraspecies resonances of Dy or Er and have no intraspecies resonances themselves, they may be used to probe the interspecies resonances, even in regions where intraspecies resonances exist. Alternatively, if the closedshell atom is in large excess, three-body losses due to the intraspecies resonances may be sufficiently suppressed to work with the interspecies resonances. In any case, there are numerous windows (up to approximately $1 \mathrm{G}$ wide) that are free from intraspecies resonances and suitable for working with interspecies resonances.

The resonance-free windows should also be ideal for evaporative cooling of both species to quantum degeneracy. The recent successful creation of dual-degenerate gases of Dy and Er [97] is extremely encouraging in this respect. Simultaneous evaporative cooling requires a favorable interspecies scattering length, which may be achieved either by tuning across a wide resonance with a magnetic field or (in the low-anisotropy case) by a suitable choice of isotopes to achieve the required background scattering length.

Once degeneracy is achieved, it should be possible to load the mixture into a 3D optical lattice. Such a lattice provides a very sensitive environment for the detection of Feshbach resonances [98] and an ideal platform for molecule formation. A lattice with only one atom of Dy or Er per site eliminates the complication of intraspecies resonances and allows magnetoassociation at any desired magnetic field.

Magnetoassociation is usually most effective at relatively narrow resonances, with widths around $100 \mathrm{mG}-1 \mathrm{G}$. Many such resonances exist in both limiting regimes of anisotropy. In the low-anisotropy case, they are mostly due to bound states with $L=4$ or 6 . The background scattering length for these resonances is moderately tunable with a magnetic field, as seen in Fig. 1, but is centered around the isotropic scattering length $a_{\text {iso }}$. If necessary, $a_{\text {iso }}$ may be selected by choosing an appropriate isotopic combination. In the high-anisotropy case, there are narrow resonances suitable for magnetoassociation, with a wide variety of background scattering lengths even for a single interaction potential (or isotopic combination). In this case, careful selection of isotopes may be unnecessary.

Transferring molecules formed by magnetoassociation to short-range (low-lying) states with electric dipole moments will be a challenge. Nevertheless, it can probably be achieved by STIRAP, as has been possible for a variety of alkali-metal dimers. Detailed spectroscopy of the intermediate electronic states available for STIRAP will be required but is outside the scope of the present paper.

The short-range states of these molecules are probably best described by Hund's case (c) [99]. Here, the atomic total angular momentum $j$ is projected onto the internuclear axis with projection $\Omega$, which can take values from $-j$ to $j$. For each value of $\Omega$, the total angular momentum $J$ can take values $J \geq|\Omega|$ in integer steps. If $j$ is a good quantum number, states $\Omega$ and $\Omega^{\prime}$ with $\Omega-\Omega^{\prime}= \pm 1$ are coupled by Coriolis matrix elements proportional to $\{[j(j+1)-$ $\left.\left.\Omega \Omega^{\prime}\right]\left[J(J+1)-\Omega \Omega^{\prime}\right]\right\}^{1 / 2}$, which ultimately uncouple $j$ from the internuclear axis at high $J$.

In a magnetic field, each state is split into $2 J+1$ components with space-fixed projection $M$ from $-J$ to $J$. The $g$ factor for Hund's case (c) is [100]

$\frac{\Omega^{2}}{2 J(J+1)}\left(g_{s}+g_{l}+\left(g_{s}-g_{l}\right) \frac{s(s+1)-l(l+1)}{j(j+1)}\right)$.

The quantity in parentheses is approximately $7 / 3$ for ${ }^{3} \mathrm{H}_{6}$ and $5 / 2$ for ${ }^{5} \mathrm{I}_{8}$. For $J=|\Omega|$, there are $2|\Omega|+1$ such states, equally spaced at low field. It should be straightforward to transfer population between these states and create coherent superpositions of them with microwave pulses, as has been achieved for alkali-metal dimers [101]. The large number of states available offer the opportunity to create fully controllable high-dimensional quantum systems, which may be used as qudits for quantum computation [18].

In zero electric field, states with positive and negative values of $\Omega$ combine to form pairs of states of opposite parity. These parity eigenstates have no permanent dipole moment. However, they are separated only by coupling to $\Omega=0$, which exists for only one parity combination. They are nearly degenerate and become more closely so with increasing $|\Omega|$. For states with high $|\Omega|$, it requires only a very small electric field to mix the parity eigenstates $(\Omega$ doublets) and form oriented states with dipole moment $\mu_{v} \Omega M /[J(J+1)]$. Here, $\mu_{v}$ is the body-fixed molecular dipole moment; $\Omega$ and $M$ are signed quantities and the space-fixed dipole depends on their relative sign. These states are analogous to those of symmetric-top molecules, which are expected to have important applications in the simulation of quantum magnetism [43,44] and in quantum computing [45].

The interactions of high-spin atoms with closed-shell atoms are substantially simpler than those with alkali-metal atoms or with themselves. As described above, the interaction of $\mathrm{Dy}\left({ }^{5} \mathrm{I}_{8}\right)$ with a closed-shell atom is characterized by at most seven potential curves, $V_{\lambda}(R)$. If the short-range anisotropy is weak, the scattering lengths for the seven curves may be related to one another; even if not, it is likely to be feasible to extract this many scattering lengths from experimental measurements on Feshbach resonances and near-threshold bound states. Interactions of high-spin atoms with alkali-metal atoms [72] require twice as many potential curves, because there are two possible spin multiplicities for each $\lambda$. Interactions of high-spin atoms with themselves require many more potential curves [66]. 


\section{CONCLUSIONS}

We have considered Feshbach resonances in collisions of an open-shell high-spin atom (Dy or Er) with a closed-shell atom $(\mathrm{Yb}$ or $\mathrm{Sr})$. We have developed model interaction potentials for $\mathrm{Dy}+\mathrm{Yb}$ and $\mathrm{Er}+\mathrm{Sr}$ and calculated scattering lengths and the positions of near-threshold bound states as a function of the magnetic field. For both systems, we have found numerous Feshbach resonances, with a variety of widths, at moderate magnetic fields.

The couplings responsible for Feshbach resonances depend on the anisotropy of the interaction potential. We have considered two limiting regimes of anisotropy. In the first limit, we consider anisotropy due entirely to dispersion forces, which arises from the tensor polarizability of the open-shell atom. This produces quite weak anisotropies in the long-range potential for atoms like Dy and Er, in which the unpaired $f$ electrons lie mostly inside the outermost $s$ electrons. The resulting interaction produces direct couplings with selection rule $\Delta L \leq 2$, where $L$ is the end-overend angular momentum of the colliding pair. In this regime, the strongest resonances in $s$-wave scattering are due to bound states with $L=2$; we show that at least one such resonance must occur below $98 \mathrm{G}$ for $\mathrm{Dy}+\mathrm{Yb}$ and below $176 \mathrm{G}$ for $\mathrm{Er}+\mathrm{Sr}$. Additional broad resonances occur at somewhat higher fields, and there are also narrower resonances due to bound states with $L>4$. In the second limit, we consider much stronger anisotropy that may exist due to higher-order dispersion forces, chemical bonding, or repulsive anisotropy. In this regime, there is much stronger coupling between different values of $L$, such that $L$ is no longer even nearly conserved. This produces many more Feshbach resonances but still with a wide variety of widths.

The two regimes of anisotropy that we have considered span the range of likely physical behavior for these systems. The long-range model represents the minimum coupling that is likely to exist, while the short-range model represents the maximum. For both models, we predict many Feshbach resonances at experimentally accessible magnetic fields, with a variety of widths. Additional resonances may occur when one or both atoms have nuclear spin.

The resonances predicted here have a wide range of possible applications. The wider resonances are very suitable for tuning interspecies interactions, both to achieve properties desirable for formation of dual-degenerate gases and to investigate the novel properties of mixtures of dipolar and nondipolar species [47,48,50]. Bose-Bose, Bose-Fermi, and Fermi-Fermi mixtures are accessible. Further tuning may be achieved by selecting from the wide variety of isotopic combinations available. The narrower resonances may be used for magnetoassociation to form high-spin molecules that inherit the large magnetic moments of Er and Dy. These may, in turn, be transferred to short-range states where they will still have large magnetic moments. In addition, there will be near-degenerate pairs of $\Omega$ doublets, of opposite parity, which will be easily mixed with very small electric fields to form oriented states with significant space-fixed electric dipole moments.

The general properties of the resonances predicted here will hold for a variety of systems. They apply to any combination of a heavy atom with high orbital angular momentum and a heavy closed-shell atom. The densities of near-threshold states and of the resulting resonances will be lower if either atom is significantly lighter, but the general considerations still apply for atoms of mass 20 or more. They apply to transition-metal atoms as well as lanthanides or actinides. They do not apply to open-shell atoms in S states or to very light atoms such as $\mathrm{He}$ and first-row elements.

In summary, we believe that this study reveals new directions for the study of strongly interacting quantum gases and opens up a new field of high-spin dipolar molecules. We have demonstrated the feasibility of producing such molecules from laser-cooled atoms and outlined some of their properties and potential applications.

The data presented in this work are available from Durham University [102].

\section{ACKNOWLEDGMENTS}

We are grateful to Alex Guttridge for valuable discussions. This work was supported by the United Kingdom Engineering and Physical Sciences Research Council (EPSRC) Grants No. EP/N007085/1, No. EP/P008275/1, and No. EP/P01058X/1.

Note added.-Recently, we learned of parallel work on the related system $\mathrm{Er}+\mathrm{Yb}$ that also predicts many resonances at low field [103].

[1] C. Chin, R. Grimm, P. S. Julienne, and E. Tiesinga, Feshbach Resonances in Ultracold Gases, Rev. Mod. Phys. 82, 1225 (2010).

[2] A. J. Moerdijk, B. J. Verhaar, and A. Axelsson, Resonances in Ultracold Collisions of ${ }^{6} \mathrm{Li},{ }^{7} \mathrm{Li}$, and ${ }^{23} \mathrm{Na}$, Phys. Rev. A 51, 4852 (1995).

[3] I. Bloch, J. Dalibard, and W. Zwerger, Many-Body Physics with Ultracold Gases, Rev. Mod. Phys. 80, 885 (2008).

[4] T. Köhler, K. Góral, and P. S. Julienne, Production of Cold Molecules via Magnetically Tunable Feshbach Resonances, Rev. Mod. Phys. 78, 1311 (2006).

[5] K.-K. Ni, S. Ospelkaus, M. H. G. de Miranda, A. Pe'er, B. Neyenhuis, J. J. Zirbel, S. Kotochigova, P. S. Julienne, D. S. Jin, and J. Ye, A High Phase-Space-Density Gas of Polar Molecules in the Rovibrational Ground State, Science 322, 231 (2008).

[6] T. Takekoshi, L. Reichsöllner, A. Schindewolf, J. M. Hutson, C. R. Le Sueur, O. Dulieu, F. Ferlaino, R. Grimm, and H.-C. Nägerl, Ultracold Dense Samples of Dipolar $\mathrm{RbCs}$ Molecules in the Rovibrational and Hyperfine Ground State, Phys. Rev. Lett. 113, 205301 (2014). 
[7] P. K. Molony, P. D. Gregory, Z. Ji, B. Lu, M. P. Köppinger, C. R. Le Sueur, C. L. Blackley, J. M. Hutson, and S. L. Cornish, Creation of Ultracold ${ }^{87} \mathrm{Rb}^{133} \mathrm{Cs}$ Molecules in the Rovibrational Ground State, Phys. Rev. Lett. 113, 255301 (2014).

[8] F. Wang, X. He, X. Li, B. Zhu, J. Chen, and D. Wang, Formation of Ultracold NaRb Feshbach Molecules, New J. Phys. 17, 035003 (2015).

[9] J. W. Park, S. A. Will, and M. W. Zwierlein, Ultracold Dipolar Gas of Fermionic ${ }^{23} \mathrm{Na}^{40} \mathrm{~K}$ Molecules in Their Absolute Ground State, Phys. Rev. Lett. 114, 205302 (2015).

[10] M. Guo, B. Zhu, B. Lu, X. Ye, F. Wang, R. Vexiau, N. Bouloufa-Maafa, G. Quéméner, O. Dulieu, and D. Wang, Creation of an Ultracold Gas of Ground-State Dipolar ${ }^{23} \mathrm{Na}^{87} \mathrm{Rb}$ Molecules, Phys. Rev. Lett. 116, 205303 (2016).

[11] A. V. Gorshkov, S. R. Manmana, G. Chen, J. Ye, E. Demler, M. D. Lukin, and A. M. Rey, Tunable Superfluidity and Quantum Magnetism with Ultracold Polar Molecules, Phys. Rev. Lett. 107, 115301 (2011).

[12] B. Yan, S. A. Moses, B. Gadway, J. P. Covey, K. R. A. Hazzard, A. M. Rey, D. S. Jin, and J. Ye, Observation of Dipolar Spin-Exchange Interactions with Lattice-Confined Polar Molecules, Nature (London) 501, 521 (2013).

[13] K. R. A. Hazzard, B. Gadway, M. Foss-Feig, B. Yan, S. A. Moses, J. P. Covey, N. Y. Yao, M. D. Lukin, J. Ye, D. S. Jin, and A. M. Rey, Many-Body Dynamics of Dipolar Molecules in an Optical Lattice, Phys. Rev. Lett. 113, 195302 (2014).

[14] F. Seeßelberg, X.-Y. Luo, M. Li, R. Bause, S. Kotochigova, I. Bloch, and C. Gohle, Extending Rotational Coherence of Interacting Polar Molecules in a Spin-Decoupled Magic Trap, Phys. Rev. Lett. 121, 253401 (2018).

[15] D. DeMille, Quantum Computation with Trapped Polar Molecules, Phys. Rev. Lett. 88, 067901 (2002).

[16] K.-K. Ni, T. Rosenband, and D. D. Grimes, Dipolar Exchange Quantum Logic Gate with Polar Molecules, Chem. Sci. 9, 6830 (2018).

[17] J. A. Blackmore, L. Caldwell, P. D. Gregory, E. M. Bridge, R. Sawant, J. Aldegunde, J. Mur-Petit, D. Jaksch, J. M. Hutson, B. E. Sauer, M. R. Tarbutt, and S. L. Cornish, Ultracold Molecules: A Platform for Quantum Simulation, Quantum Sci. Technol. 4, 014010 (2019).

[18] R. Sawant, J. A. Blackmore, P. D. Gregory, J. Mur-Petit, D. Jaksch, J. Aldegunde, J. M. Hutson, M. R. Tarbutt, and S. L. Cornish, Ultracold Molecules as Qudits, New J. Phys. 22, 013027 (2020).

[19] N. Balakrishnan and A. Dalgarno, Chemistry at Ultracold Temperatures, Chem. Phys. Lett. 341, 652 (2001).

[20] T. V. Tscherbul and R. V. Krems, Manipulating SpinDependent Interactions in Rotationally Excited Cold Molecules with Electric Fields, J. Chem. Phys. 125, 194311 (2006).

[21] M.-G. Hu, Y. Liu, D. Grimes, Y.-W. Lin, A. Gheorghe, R. Vexiau, N. Bouloufa-Maafa, O. Dulieu, T. Rosenband, and K.-K. Ni, Direct Observation of Bimolecular Reactions of Ultracold KRb Molecules, Science 366, 1111 (2019).

[22] A. Micheli, G. K. Brennen, and P. Zoller, A Toolbox for Lattice-Spin Models with Polar Molecules, Nat. Phys. 2, 341 (2006).
[23] M. A. Baranov, M. Dalmonte, G. Pupillo, and P. Zoller, Condensed Matter Theory of Dipolar Quantum Gases, Chem. Rev. 112, 5012 (2012).

[24] M. S. Safronova, D. Budker, D. DeMille, De. F. J. Kimball, A. Derevianko, and C. W. Clark, Search for New Physics with Atoms and Molecules, Rev. Mod. Phys. 90, 025008 (2018).

[25] D. J. McCarron, M. H. Steinecker, Y. Zhu, and D. DeMille, Magnetic Trapping of an Ultracold Gas of Polar Molecules, Phys. Rev. Lett. 121, 013202 (2018).

[26] L. Anderegg, B. L. Augenbraun, Y. Bao, S. Burchesky, L. W. Cheuk, W. Ketterle, and J. M. Doyle, Laser Cooling of Optically Trapped Molecules, Nat. Phys. 14, 890 (2018).

[27] A. L. Collopy, S. Ding, Y. Wu, I. A. Finneran, L. Anderegg, B. L. Augenbraun, J. M. Doyle, and J. Ye, $3 D$ Magneto-Optical Trap of Yttrium Monoxide, Phys. Rev. Lett. 121, 213201 (2018).

[28] L. Caldwell, J. A. Devlin, H. J. Williams, N. J. Fitch, E. A. Hinds, B. E. Sauer, and M. R. Tarbutt, Deep Laser Cooling and Efficient Magnetic Compression of Molecules, Phys. Rev. Lett. 123, 033202 (2019).

[29] P. S. Żuchowski, J. Aldegunde, and J. M. Hutson, Ultracold RbSr Molecules Can be Formed by Magnetoassociation, Phys. Rev. Lett. 105, 153201 (2010).

[30] F. Münchow, C. Bruni, M. Madalinski, and A. Görlitz, Two-Photon Photoassociation Spectroscopy of Heteronuclear YbRb, Phys. Chem. Chem. Phys. 13, 18734 (2011).

[31] D. A. Brue and J. M. Hutson, Prospects of Forming Molecules in ${ }^{2} \Sigma$ States by Magnetoassociation of Alkali-Metal Atoms with Yb, Phys. Rev. A 87, 052709 (2013).

[32] A. Guttridge, M. D. Frye, B. C. Yang, J. M. Hutson, and S. L. Cornish, Two-Photon Photoassociation Spectroscopy of CsYb: Ground-State Interaction Potential and Interspecies Scattering Lengths, Phys. Rev. A 98, 022707 (2018).

[33] V. Barbé, A. Ciamei, B. Pasquiou, L. Reichsöllner, F. Schreck, P. S. Żuchowski, and J. M. Hutson, Observation of Feshbach Resonances between Alkali and Closed-Shell Atoms, Nat. Phys. 14, 881 (2018).

[34] B. C. Yang, M. D. Frye, A. Guttridge, J. Aldegunde, P. S. Żuchowski, S. L. Cornish, and J. M. Hutson, Magnetic Feshbach Resonances in Ultracold Collisions between Cs and Yb Atoms, Phys. Rev. A 100, 022704 (2019).

[35] Y. Takasu, K. Maki, K. Komori, T. Takano, K. Honda, M. Kumakura, T. Yabuzaki, and Y. Takahashi, Spin-Singlet Bose-Einstein Condensation of Two-Electron Atoms, Phys. Rev. Lett. 91, 040404 (2003).

[36] T. Fukuhara, S. Sugawa, Y. Takasu, and Y. Takahashi, AllOptical Formation of Quantum Degenerate Mixtures, Phys. Rev. A 79, 021601(R) (2009).

[37] S. Stellmer, M. K. Tey, B. Huang, R. Grimm, and F. Schreck, Bose-Einstein Condensation of Strontium, Phys. Rev. Lett. 103, 200401 (2009).

[38] M. Lu, N. Q. Burdick, S. H. Youn, and B. L. Lev, Strongly Dipolar Bose-Einstein Condensate of Dysprosium, Phys. Rev. Lett. 107, 190401 (2011).

[39] M. Lu, N. Q. Burdick, and B. L. Lev, Quantum Degenerate Dipolar Fermi Gas, Phys. Rev. Lett. 108, 215301 (2012). 
[40] K. Aikawa, A. Frisch, M. Mark, S. Baier, A. Rietzler, R. Grimm, and F. Ferlaino, Bose-Einstein Condensation of Erbium, Phys. Rev. Lett. 108, 210401 (2012).

[41] K. Aikawa, A. Frisch, M. Mark, S. Baier, R. Grimm, and F. Ferlaino, Reaching Fermi Degeneracy via Universal Dipolar Scattering, Phys. Rev. Lett. 112, 010404 (2014).

[42] H. L. Bethlem and G. Meijer, Production and Application of Translationally Cold Molecules, Int. Rev. Phys. Chem. 22, 73 (2003).

[43] M. L. Wall, K. Maeda, and L. D. Carr, Simulating Quantum Magnets with Symmetric Top Molecules, Ann. Phys. (Amsterdam) 525, 845 (2013).

[44] M. L. Wall, K. Maeda, and L. D. Carr, Realizing Unconventional Quantum Magnetism with Symmetric Top Molecules, New J. Phys. 17, 025001 (2015).

[45] P. Yu, L. W. Cheuk, I. Kozyryev, and J. M. Doyle, A Scalable Quantum Computing Platform Using SymmetricTop Molecules, New J. Phys. 21, 093049 (2019).

[46] K. Zaremba-Kopczyk, P. S. Żuchowski, and M. Tomza, Magnetically Tunable Feshbach Resonances in Ultracold Gases of Europium Atoms and Mixtures of Europium and Alkali-Metal Atoms, Phys. Rev. A 98, 032704 (2018).

[47] B. Capogrosso-Sansone, Solid Phases and Pairing in a Mixture of Polar Molecules and Atoms, Phys. Rev. A 83, 053611 (2011).

[48] L. E. Young-S. and S. K. Adhikari, Dipolar Droplet Bound in a Trapped Bose-Einstein Condensate, Phys. Rev. A 87, 013618 (2013).

[49] K. B. Gubbels and H. T. C. Stoof, Imbalanced Fermi Gases at Unitarity, Phys. Rep. 525, 255 (2013).

[50] J. E. Baarsma and P. Törmä, Fermi Surface Deformations and Pairing in Mixtures of Dipolar and Non-dipolar Fermions, arXiv:1612.07953.

[51] C. Ravensbergen, V. Corre, E. Soave, M. Kreyer, E. Kirilov, and R. Grimm, Production of a Degenerate Fermi-Fermi Mixture of Dysprosium and Potassium Atoms, Phys. Rev. A 98, 063624 (2018).

[52] For both operators and quantum numbers, we follow the common collisional convention of using lowercase letters for individual atoms and uppercase letters for the interacting pair.

[53] W. C. Martin, R. Zalubas, and L. Hagan, Atomic Energy Levels-The Rare-Earth Elements. The Spectra of Lanthanum, Cerium, Praseodymium, Neodymium, Promethium, Samarium, Europium, Gadolinium, Terbium, Dysprosium, Holmium, Erbium, Thulium, Ytterbium, and Lutetium, technical report, National Standard Reference Data System, 1978.

[54] A. Stein, H. Knöckel, and E. Tiemann, The ${ }^{1} \mathrm{~S}+{ }^{1} \mathrm{~S}$ Asymptote of $\mathrm{Sr}_{2}$ Studied by Fourier-Transform Spectroscopy, Eur. Phys. J. D 57, 171 (2010).

[55] M. Borkowski, A. A. Buchachenko, R. Ciuryło, P. S. Julienne, H. Yamada, Y. Kikuchi, K. Takahashi, Y. Takasu, and Y. Takahashi, Beyond-Born-Oppenheimer Effects in Sub-kHz-Precision Photoassociation Spectroscopy of Ytterbium Atoms, Phys. Rev. A 96, 063405 (2017).

[56] S. G. Porsev, M. S. Safronova, A. Derevianko, and C. W. Clark, Long-Range Interaction Coefficients for Ytterbium Dimers, Phys. Rev. A 89, 012711 (2014).
[57] M. S. Safronova, S. G. Porsev, and C. W. Clark, Ytterbium in Quantum Gases and Atomic Clocks: van der Waals Interactions and Blackbody Shifts, Phys. Rev. Lett. 109, 230802 (2012).

[58] M. Lepers, J.-F. Wyart, and O. Dulieu, Anisotropic Optical Trapping of Ultracold Erbium Atoms, Phys. Rev. A 89, 022505 (2014).

[59] H. Li, J.-F. Wyart, O. Dulieu, S. Nascimbène, and M. Lepers, Optical Trapping of Ultracold Dysprosium Atoms: Transition Probabilities, Dynamic Dipole Polarizabilities and van der Waals $C_{6}$ Coefficients, J. Phys. B 50, 014005 (2016).

[60] K. T. Tang, Dynamic Polarizabilities and van der Waals Coefficients, Phys. Rev. 177, 108 (1969).

[61] P. Schwerdtfeger and J. K. Nagle, 2018 Table of Static Dipole Polarizabilities of the Neutral Elements in the Periodic Table, Mol. Phys. 117, 1200 (2019).

[62] X. Chu, A. Dalgarno, and G. C. Groenenboom, Dynamic Polarizabilities of Rare-Earth-Metal Atoms and Dispersion Coefficients for Their Interaction with Helium Atoms, Phys. Rev. A 75, 032723 (2007).

[63] A. Stein, H. Knöckel, and E. Tiemann, Fourier-Transform Spectroscopy of $\mathrm{Sr}_{2}$ and Revised Ground-State Potential, Phys. Rev. A 78, 042508 (2008).

[64] P. Tecmer, K. Boguslawski, M. Borkowski, P. S. Żuchowski, and D. Kędziera, Modeling the Electronic Structures of the Ground and Excited States of the Ytterbium Atom and the Ytterbium Dimer: A Modern Quantum Chemistry Perspective, Int. J. Quantum Chem. 119, e25983 (2019).

[65] M. Kitagawa, K. Enomoto, K. Kasa, Y. Takahashi, R. Ciuryło, P. Naidon, and P. S. Julienne, Two-Color Photoassociation Spectroscopy of Ytterbium Atoms and the Precise Determinations of $s$-Wave Scattering Lengths, Phys. Rev. A 77, 012719 (2008).

[66] A. Petrov, E. Tiesinga, and S. Kotochigova, AnisotropyInduced Feshbach Resonances in a Quantum Dipolar Gas of Highly Magnetic Atoms, Phys. Rev. Lett. 109, 103002 (2012).

[67] J. M. Hutson, Theory of Cold Atomic and Molecular Collisions, in Cold Molecules: Theory, Experiment, Applications, edited by R. V. Krems, W. C. Stwalley, and B. Friedrich (Taylor \& Francis, London, 2009), pp. 3-37.

[68] J. M. Hutson and C. R. Le Sueur, MOLSCAT, BOUND and FIELD, version 2020.0 (2020), https://github.com/molscat/ molscat.

[69] J. M. Hutson and C. R. Le Sueur, MoLSCAT: A Program for Non-reactive Quantum Scattering Calculations on Atomic and Molecular Collisions, Comput. Phys. Commun. 241, 9 (2019).

[70] J. M. Hutson and C. R. Le Sueur, BOUND and FIELD: Programs for Calculating Bound States of Interacting Pairs of Atoms and Molecules, Comput. Phys. Commun. 241, 1 (2019).

[71] M. L. González-Martínez and J. M. Hutson, Magnetically Tunable Feshbach Resonances in $\mathrm{Li}+\mathrm{Yb}\left({ }^{3} P_{J}\right)$, Phys. Rev. A 88, 020701(R) (2013).

[72] M. L. González-Martínez and P.S. Żuchowski, Magnetically Tunable Feshbach Resonances in $\mathrm{Li}+\mathrm{Er}$, Phys. Rev. A 92, 022708 (2015). 
[73] M. L. González-Martínez and J. M. Hutson, Sympathetic Cooling of Fluorine Atoms with Ultracold Atomic Hydrogen, Phys. Rev. A 88, 053420 (2013).

[74] D. E. Manolopoulos, An Improved Log-Derivative Method for Inelastic Scattering, J. Chem. Phys. 85, 6425 (1986).

[75] D. E. Manolopoulos, M. J. Jamieson, and A. D. Pradhan, Johnson's Log Derivative Algorithm Rederived, J. Comput. Phys. 105, 169 (1993).

[76] M. H. Alexander, Hybrid Quantum Scattering Algorithms for Long-Range Potentials, J. Chem. Phys. 81, 4510 (1984).

[77] M. H. Alexander and D. E. Manolopoulos, A Stable Linear Reference Potential Algorithm for Solution of the Quantum Close-Coupled Equations in Molecular Scattering Theory, J. Chem. Phys. 86, 2044 (1987).

[78] J. M. Hutson, Coupled-Channel Methods for Solving the Bound-State Schrödinger Equation, Comput. Phys. Commun. 84, 1 (1994).

[79] M. D. Frye and J. M. Hutson, Characterizing Feshbach Resonances in Ultracold Scattering Calculations, Phys. Rev. A 96, 042705 (2017).

[80] M. D. Frye and J. M. Hutson, Characterizing Quasibound States and Scattering Resonances, Phys. Rev. Research 2, 013291 (2020).

[81] G. F. Gribakin and V. V. Flambaum, Calculation of the Scattering Length in Atomic Collisions Using the Semiclassical Approximation, Phys. Rev. A 48, 546 (1993).

[82] See Supplemental Material at [http://link.aps.org/ supplemental/10.1103/PhysRevX.10.041005] for plots of scattering lengths, bound states, and Feshbach resonance statistics for $\mathrm{Er}+\mathrm{Sr}$ and on additional interaction potentials for $\mathrm{Dy}+\mathrm{Yb}$.

[83] B. Gao, Zero-Energy Bound or Quasibound States and Their Implications for Diatomic Systems with an Asymptotic van der Waals Interaction, Phys. Rev. A 62, 050702 (R) (2000).

[84] J. M. Hutson, Feshbach Resonances in the Presence of Inelastic Scattering: Threshold Behavior and Suppression of Poles in Scattering Lengths, New J. Phys. 9, 152 (2007).

[85] Z. Idziaszek and P. S. Julienne, Universal Rate Constants for Reactive Collisions of Ultracold Molecules, Phys. Rev. Lett. 104, 113202 (2010).

[86] M. D. Frye, P. S. Julienne, and J. M. Hutson, Cold Atomic and Molecular Collisions: Approaching the Universal Loss Regime, New J. Phys. 17, 045019 (2015).

[87] S. Kato, S. Sugawa, K. Shibata, R. Yamamoto, and Y. Takahashi, Control of Resonant Interaction between Electronic Ground and Excited States, Phys. Rev. Lett. 110, 173201 (2013).

[88] D. A. Brue and J. M. Hutson, Magnetically Tunable Feshbach Resonances in Ultracold Li-Yb Mixtures, Phys. Rev. Lett. 108, 043201 (2012).
[89] W. J. Childs, Hyperfine Structure of ${ }^{5} \mathrm{I}_{7,8}$ Atomic States of Dy ${ }^{161,163}$ and the Ground-State Nuclear Moments, Phys. Rev. A 2, 1692 (1970).

[90] W. J. Childs, L. S. Goodman, and V. Pfeufer, Hyperfine Structure of the $4 f^{12} 6 s^{2}{ }^{3} \mathrm{H}$ and ${ }^{3} \mathrm{~F}$ Terms of ${ }^{167} \mathrm{Er} I$ by Atomic-Beam, Laser-rf Double Resonance, Phys. Rev. A 28, 3402 (1983).

[91] M. Tomza, Prospects for Ultracold Polar and Magnetic Chromium-Closed-Shell-Atom Molecules, Phys. Rev. A 88, 012519 (2013).

[92] M. Tomza, Ab Initio Properties of the Ground-State Polar and Paramagnetic Europium-Alkali-Metal-Atom and Europium-Alkaline-Earth-Metal-Atom Molecules, Phys. Rev. A 90, 022514 (2014).

[93] T. Fukuhara, S. Sugawa, and Y. Takahashi, Bose-Einstein Condensation of an Ytterbium Isotope, Phys. Rev. A 76, 051604(R) (2007).

[94] A. Frisch, M. Mark, K. Aikawa, F. Ferlaino, J. L. Bohn, C. Makrides, A. Petrov, and S. Kotochigova, Quantum Chaos in Ultracold Collisions of Gas-Phase Erbium Atoms, Nature (London) 507, 475 (2014).

[95] K. Baumann, N. Q. Burdick, M. Lu, and B. L. Lev, Observation of Low-Field Fano-Feshbach Resonances in Ultracold Gases of Dysprosium, Phys. Rev. A 89 , 020701(R) (2014).

[96] T. Maier, H. Kadau, M. Schmitt, M. Wenzel, I. FerrierBarbut, T. Pfau, A. Frisch, S. Baier, K. Aikawa, L. Chomaz, M. J. Mark, F. Ferlaino, C. Makrides, E. Tiesinga, A. Petrov, and S. Kotochigova, Emergence of Chaotic Scattering in Ultracold Er and Dy, Phys. Rev. X 5, 041029 (2015).

[97] A. Trautmann, P. Ilzhöfer, G. Durastante, C. Politi, M. Sohmen, M. J. Mark, and F. Ferlaino, Dipolar Quantum Mixtures of Erbium and Dysprosium Atoms, Phys. Rev. Lett. 121, 213601 (2018).

[98] M. J. Mark, F. Meinert, K. Lauber, and H.-C. Nägerl, Mott-Insulator-Aided Detection of Ultra-narrow Feshbach Resonances, SciPost Phys. 5, 55 (2018).

[99] L. Veseth, Hund's Coupling Case (c) in Diatomic Molecules. I. Theory, J. Phys. B 6, 1473 (1973).

[100] M. McDonald, High Precision Optical Spectroscopy and Quantum State Selected Photodissociation of Ultracold ${ }^{88} \mathrm{Sr}_{2}$ Molecules in an Optical Lattice (Springer Nature, Cham, Switzerland, 2018).

[101] P. D. Gregory, J. Aldegunde, J. M. Hutson, and S. L. Cornish, Controlling the Rotational and Hyperfine State of Ultracold ${ }^{87} \mathrm{Rb}^{133} \mathrm{Cs}$ Molecules, Phys. Rev. A 94, 041403(R) (2016).

[102] M. D. Frye, S. L. Cornish, and J. M. Hutson, Supporting Data for "Prospects of Forming High-Spin Polar Molecules from Ultracold Atoms," https://doi.org/10.15128/ r1kh04dp733.

[103] M. B. Kosicki, M. Borkowski, and P. S. Żuchowski, Quantum Chaos in Feshbach Resonances of the ErYb System, New J. Phys. 22, 023024 (2020). 